Heinz-Dieter Pohl* $\quad$ UDK [811.112.2'373.45:811.163.6]:641.5(436.5)

Universität Klagenfurt DOI: 10.4312/linguistica.59.1.253-265

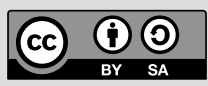

\title{
DEUTSCH-SLOWENISCHE WECHSELBEZIEHUNGEN IN DER SPRACHE DER TRADITIONELLEN KÄRNTNER KÜCHE
}

\section{EINLEITUNG}

Deutsch-slowenische Sprachkontakte finden im südlichsten österreichischen Bundesland Kärnten seit vielen Jahrhunderten statt. Seit Beginn der Landesgeschichte, als das Herzogtum Kärnten (im Jahre 976) errichtet wurde, sind sowohl Slowenen als auch Deutsche die autochthone Bevölkerung; allerdings breitet(e) sich das Deutsche bis zum heutigen Tag auf Kosten des Slowenischen immer mehr aus. Auf der Ebene der alltäglichen Umgangssprache und der Mundarten beruht die Beeinflussung der beiden Sprachen durchaus auf Gegenseitigkeit, wenngleich das Einwirken des Deutschen auf das Slowenische weitaus stärker ist bzw. in den letzten Jahrzehnten noch zugenommen hat, nicht zuletzt eine Folge der Prädominanz der deutschen Sprache - dennoch hat das Deutsche im gemischtsprachigen Gebiet auf der Ebene der Umgangssprache und der Mundarten einen slowenischen „Touch“ erhalten. Somit ist diese „Gegenseitigkeit“ sowohl in der Namengebung (v.a. Familien- und Ortsnamen) als auch in der Volkskultur deutlich feststellbar wie u.a. auch im Brauchtum und in der Küche. Im Folgenden werden rund 16 traditionell Kärntner Speisen mit ihren Bezeichnungen in beiden Sprachen vorgestellt und hinsichtlich ihrer Herkunft erklärt.

\section{REIN(D)LING UND $\check{S} A R T E L J / \check{S} A R K E L J$}

Eine der bekanntesten Kärntner Speisen ist der Reindling (so meist geschrieben nach der Aussprache) oder historisch richtig Reinling. Er ist nach der Rein(e) 'runde Schüssel oder Kasserolle; rundes, niederes Koch- oder Backgefäß; randhohe, irdene Pfanne' so benannt (Miklau 1964: 158) ${ }^{1}$ und das Kärntner Pendant zum Wiener Gugelhupf, wenn sich auch Herstellungsweise und Form auseinanderentwickelt haben. Im Gegensatz zur typischen Gugelhupfform hat der „echte“ Kärntner Reindling kein Loch in der Mitte (vgl. Miklau 1964: 93). Er wird aus feinem Germ- bzw. Hefeteig (mit Ei) hergestellt, der ausgerollt, mit Zucker oder Honig, Rosinen und Zimt bestreut und wieder zusammengerollt, in die Reine (oder auch Gugelhupfform) eingelegt und gebacken wird. ${ }^{2}$ Der (alte) Wiener Gugelhupf wurde ganz ähnlich hergestellt, allerdings nicht ausgerollt und gefüllt, sondern es wurden nur Rosinen unter den Teig

heinz.pohl@chello.at

1 Zum Lautlichen Kranzmayer (1949: 454).

2 Rezepte s. bei Miklau (1964: 93) oder Setz (1993: 91). 
gemischt und dieser wurde dann in der mit Mandelsplittern ausgelegten Gugelhupfform im Backrohr gebacken (Maier-Bruck 1984: 608). ${ }^{3}$

Der Reindling ist vom Lienzer Becken bis ins Südburgenland verbreitet; seine westliche Ausdehnung stimmt mit dem mundartlichen Merkmal der „Kärntner Dehnung“ überein (Kranzmayer 1949: 454). Der Vorläufer des "Reindlings“ war das Schartl ('Schärtlein'), so heute noch in Rückzugsgebieten der Steiermark (Kranzmayer 1949: 455). Dieses Wort ist deshalb interessant, da es einer der slowenischen Bezeichnungen des Reindlings, šartelj oder šarkelj, zugrundeliegt, z. B. dem Mießtaler koroški šarkelj 'Kärntner Reindling' (Angerer 1997: 132) oder dem Gailtaler šartelj (Grundrezept ähnlich den vorherigen, alternativ auch mit Nussfülle, so Angerer 1997: 219, Bezlaj 2005: 11f.; vgl. weiters Striedter-Temps 1963: 216 mit Lit.). Dieses Wort geht auf mittelhochdeutsch scharte 'Röstpfanne' zurück, leitet sich also ebenfalls vom Kochgeschirr ab. Schärtel bzw. Schärtling, in Kärnten auch Schartling, sind heute abgekommene Wörter für den alten (gemeinbairisch-süddeutschen) Gugelhupf. ${ }^{4}$

\section{POGAČA UND POGATSCHERL}

Ein anderes slowenisches Wort für den 'Reindling' ist pogača (Bezlaj 1995: 73), so z. B. im Rosental (ähnlich wie der Kärntner Reindling zubereitet, Fülle mit Nüssen, Rosinen, Zucker, Vanillezucker und Rum oder Variante mit Karobemehl, Zimt, gehackten Feigen und Rum, so Angerer 1997: 177) oder auf der Sattnitz jabolčna pogača 'Apfelreindling' (mit Fülle aus geschnittenen Äpfeln mit Zimt und Zucker, so (Angerer 1997: 50). ${ }^{5}$ Dieses Wort - in der slowenischen Mundart meist pohača gesprochen - ist eine Entlehnung aus romanisch focatialfocacea bzw. mittellateinisch focantia, und bezeichnete früher eine Art Weißbrot, vgl. in Tirol Fochaze 'eine Brotart aus Weizenmehl ohne Germ bzw. Hefe zu bestimmten Zeiten gebacken' (Schatz 1993: 182). In die deutschen Mundarten Kärntens ist das Wort als Fochenze eingegangen, zuletzt nur mehr in Oberkärnten gebräuchlich (Kranzmayer 1949: 456), schon seit althochdeutscher Zeit belegt, z. B. vochanza (Kloster Mondsee). Miklau (1964: 92 u. 155) erklärt das Wort als 'Osterbrot mit eingebackenen Trockenfrüchten und Nüssen' und bietet ein Rezept, das auch den Namen „Kärntner Kletzenbrot“ hat.

Im Deutschen ist heute nur die slowenische Lautung Pogatsche(n) oder Pohatscha gebräuchlich. Übrigens wird seit einigen Jahren in Ferlach/Borovlje ein Pohača-Fest veranstaltet, das sich auch bei der deutschsprachigen Bevölkerung großer Beliebtheit erfreut. Ein Diminutiv zu diesem Wort, Pogatscherl, gibt es auch in der Wiener Küche:

3 Heute versteht man in Wien unter Gugelhupf eher einen ohne Germ hergestellten Kuchen, wie er im Binnendeutschen als „Napfkuchen“ bekannt ist, entweder mit Mandeln und Rosinen oder als „Marmorgugelhupf“, indem vor dem Backen ein Drittel des Teiges mit Kakao oder Kochschokolade (ohne Rosinen) vermischt wird, um das „marmorierte“ Muster beim Anschnitt zu erzeugen (Kofranek 1975: 415f.).

4 Dieser ist nach Zehetner (2005: 162f. ein „Satzname“ nach dem Wunsch Gugel, hupf! 'Gugel (= gewölbtes, gerundetes Ding) spring heraus (aus der Form)', eventuell auch dissimiliert aus Gugel-gupf (Gupf'sich nach oben Wölbendes, höchster Punkt').

5 In Tschachoritsch/Čahorče erhoben. 
Grammelpogatscherl. Dies ist ein Weingebäck, das es sowohl in einer pikanten (mit Salz und Kümmel) als auch süßen Variante gibt (vgl. Pohl 2007: 116 mit Lit.).

\section{MEISCHEL/MEISCHERL UND MAJŽELJ}

Eine weitere slowenische Bezeichnung für den 'Reindling' ist majželj (vgl. Bezlaj 1982: 173 s. v. maželj), worauf im Deutschen auch die Maischeln oder Maischerln 'Netzlaibchen' zurückgehen (in anderen Gegenden Kärntens auch Leberlan genannt). Auf den ersten Blick scheint es recht merkwürdig, dass eine Süß- und eine Fleischspeise mit dem gleichen Wort bezeichnet werden, doch die Lösung liegt im Grundwort, das ursprünglich 'Schnitte' bedeutet hat (s. u.). Im Gailtal finden wir drei majželj genannte Speisen, den 'Festtagsreindling' majželj mit Fülle aus Rosinen, Zimt, Nüssen, Rum, Vanillezucker und Anis (Angerer 1997: 221), den 'Eierreindling' jajčni majželj mit Teig, wie er auch für Faschingskrapfen verwendet wird (Angerer 1997: 220) und 'Biestmaischeln' mlezivni majželjni, eine Hauptspeise aus Biestmilch ${ }^{6}$ (mit Weizenmehl, Eiern, Salz und Anis (Angerer 1997: 211).

Im deutschen Sprachgebrauch sind die Maischerln nur als Hauptspeise (obligater Bestandteil der „Schlachtplatte“) bekannt, zubereitet aus Schweinskopf und faschiertem Beuschel mit Herz, vermischt mit Rollgerste (oder Hirsebrein) und reichlich Gewürz. ${ }^{7}$ Die so entstandene Masse wird in das Netz eingerollt und gebraten. Im Slowenischen begegnet das Wort mit dieser Bedeutung in mehreren Varianten, auf der Sattnitz mavželj, ebenso im Rosen- und Mießtal (Angerer 1997: 38, 131 u. 171 mit Rezepten); im Gailtal in der Verkleinerungsform ${ }^{8}$ majžlč (Angerer 1997: 213) und im Kanaltal als maželj (Angerer 1997: 233). Das slowenische Wort majželj usw. ist aus altem bairischen *meisili, Verkleinerungsform zu Maise(n) 'Schnitte', entlehnt (vgl. Striedter-Temps 1963: 174 mit Lit. sowie Bezlaj 1982: 173 s. v. maželj) und ins Deutsche als Maischel (-ale) bzw. Maischerl rückentlehnt worden.

\section{PFANNZELTEN UND FANCELJ}

Unseren Fleischlaibchen (oder mundartnah Fleischlaberln) entsprechen in Bayern die Fleischpflanzeln (im nördlichen Deutschland meist Frikadellen, in und um Berlin Buletten genannt, im Südwesten Fleischküchle, neben weiteren Bezeichnungen (vgl. Seibicke 1983: 80 mit Karte). Doch diesem bayerischen Pflanzel liegt ein älteres Pfanzel zugrunde, das selbst ein gekürztes Pfannzelte 'Pfannkuchen, in der Pfanne Gebackenes u. dgl.' ist (vgl. Zehetner 2005: 263 u. 265). In älteren Kärntner Kochbüchern kommt dieses Wort ebenfalls vor, so z. B. Blutpfanzl (Pfannengericht aus Blutwurstmasse, Miklau 1964: 36) oder Hadn- bzw. Türkenpfanzl (aus Buchweizen- oder Maismehl, Miklau 1964: 70f.). Statt Pfanzl (auch Pflanzl wie in Bayern!) wird auch Tomele

6 Unter Biestmilch (auch Kolostralmilch, slowenisch mlezivo) versteht man die Erstmilch oder Vormilch. Angerer (1997: 211 u. 1998: 176) schreibt mlezvni majželjni.

7 Die genaue Rezeptur s. bei Miklau (1964: 37) und Setz (1993: 57f.), bei Maier-Bruck (1984: 88) 'Netzlaibchen' genannt.

8 Das Grundwort majželj ist ja schon für den 'Reindling' (s. o.) vergeben. 
verwendet. So verschieden also die Fleischlaibchen und -pflanzeln auf den ersten Blick auch sein mögen - das Pfanzel ist ein altes gemeinbairisches Wort. Auch hier ist das Slowenische ein Zeuge alter sprachlicher Verhältnisse. Während im österreichischen Deutschen das Wort längst aus dem modernen Wortschatz verschwunden ist, haben es die Kärntner Slowenen bis heute konserviert, als Süßspeise z. B. flancati 'Strauben' (Sattnitz, Angerer 1997: 43f.) oder piškotni fancelj 'Pfannzelten' (aus Eiern, Mehl, Salz und Butter gebacken und danach aufgeschnitten, eine Art Suppeneinlage, Jauntal, Angerer 1997: 109). In den slowenischen Mundarten kommt das Wort in mehreren Varianten vor: fancelt, fancelj, f(l)ancat usw. (Striedter-Temps 1963: 114 mit Lit.), dem ein altes, mittelhochdeutsches phan-zëlte 'Pfannenkuchen' zugrundeliegt, das zu bairisch Pfänzlein umgeformt wurde. Das - $l$ - im jüngeren Pflanzel ist sekundär, wohl in Anlehnung an Pflanze (man vgl. auch die slowenische Variante flancat mit -l-).

\section{KRAPFEN UND KRAPI}

Unter einem Krapfen versteht man (auch in Bayern und überhaupt im süddeutschen Raum) heute in erster Linie ein Süßgebäck aus Germ- bzw. Hefeteig, meist mit Marmelade gefüllt, so als 'Faschingskrapfen' v.a. in Wien oder als 'Bauernkrapfen' (auch in Fladen- oder Radform, mehrere Rezepte bei Setz 1993: 76ff.) in Kärnten allgemein bekannt. Doch die alte Bedeutung des Wortes Krapfen war eine andere, dies zeigt schon die Etymologie, mittelhochdeutsch krapfe 'Haken'. Waren die Vorläufer der heutigen Krapfen hakenförmig? Oder wurden sie aus der Teigmasse mit hakenförmigem Gerät ,ausgestochen“, bevor sie in die Pfanne kamen? Die etymologischen Wörterbücher geben leider keine Auskunft. Wie dem auch sei: das Endprodukt muss auf jeden Fall ein 'Krapfen', etwas Rundes, Bauchiges, gewesen sein. Runde Teigspeisen sind aber nicht nur Germ- bzw. Hefegebäcke, sondern auch gefüllte Nudeln, und hier schließt sich der Kreis. In Tirol (und Oberkärnten ${ }^{9}$ ) ist Krapfen noch heute zweideutig:

1. (süßes) Germ- bzw. Hefegebäck;

2. (gefülltes und gesottenes) Nudelgericht.

Was dem Kärntner seine „Käsnudeln“, sind dem Tiroler seine „Schlutz- oder Schlipfkrapfen" (mit verschiedenen Füllungen, meist Kartoffeln, Topfen oder Spinat). An die in Tirol verbreitete Bedeutung erinnern heute noch in Kärnten die Schlickkrapferln, der „kleine Bruder“ der Kärntner Fleischnudeln als Suppeneinlage. Sie werden entweder aus Fleischresten oder aus gekochtem und faschiertem Beuschel (Lunge), mit Gewürzen, Ei und Semmelbröseln hergestellt; sie heißen in Oberkärnten auch Schlutzkrapfen (vgl. Setz 1993: 10f.).

Bei den Kärntner Slowenen ist der alte Bedeutungsumfang von 'Krapfen' im Lehnwort krap, Plural krapi, noch immer lebendig. Das Kärntner „Nationalgericht“ Käsnudeln heißt auf Slowenisch sirnati krapi 'Käsekrapfen' (Sattnitz, Rosental, Angerer 1997: 22 u. 140), im Gailtal ziljski krapi oder einfach krapi (Angerer 1997: 200f.); daneben gibt es freilich auch mesni krapi 'Fleischnudeln' (Angerer 1997: 206), čompavi

9 Z. B. Oberdrauburg (nach eigenen Erhebungen). 
krapi 'Erdäpfelnudeln' (Angerer 1997: 199 - das typisch gailtalerische Wort für 'Kartoffel', čompe, enthaltend, auch in die deutsche Mundart als Tschompe entlehnt), skutni krapi 'Topfennudeln' (Angerer 1997: 254), sowie als Süßspeise ščipni krapi 'Germnudeln' und v. a. kvočni krapi ${ }^{10}$ 'Kletzennudeln' (Rosental, Angerer 1997: 142f.).

Ganz andere Krapfen sind die suhi krapi 'trockene Krapfen' im Jauntal (Angerer 1997: 105), die eine weitere alte Bedeutung, nämlich 'Fladengebäck', reflektieren. Die typische Krapfenform haben aber nur Germ- bzw. Hefeteige; wo das Wort Krapfen nicht üblich ist, steht dafür Pfannkuchen, worunter wir aber heute v. a. Palatschinken verstehen, ${ }^{11}$ für den Norddeutschen können allerdings Pfannkuchen auch 'Krapfen' sein, im Nordwesten und Südwesten meist Berliner genannt, abgekürzt aus Berliner Pfannkuchen. ${ }^{12}$

Die eingangs erwähnten Schlickkrapferln scheinen auf Schlittkrapfen zurückzugehen, die ursprüngliche Bedeutung war 'gefüllter und gesottener Krapfen (Suppeneinlage aus Fleisch mit Teighülle)'; die genaue Herkunft ist nicht bekannt. Sie sind ins Slowenische als žli(n)krofi (Plural) entlehnt worden. Neben den typisch kärntnerischen Schlickkrapferln (Miklau 1964: 41 u. Setz 1993: 11) gibt es noch mehrere andere Speisen, die dieses Wort enthalten, z. B. ajdovi žlinkrofi 'Heiden-Schlickkrapfen' (mit Rahm-Butter-Heidenmehlfüllung, ungezuckert mit Salat, gezuckert mit Apfelkompott serviert, Mießtal, Angerer 1997: 124).

\section{POTITZE UND SLOWENISCH POTICA}

Dies ist eine v. a. in Kärnten und der Steiermark verbreitete Mehlspeise, ein Rollkuchen (Germ- bzw. Hefeteig) mit Mohn- bzw. Nussfülle. Daher die Bezeichnungen Mohn- und Nusspotitze. Das Wort beruht auf slowenisch potica, mundartlich auch povtica aus älterem povitica 'Eingerolltes' im Sinne von Bäckerei, Strudel usw. (Pohl 2004: 62, Snoj 2003: 552).

\section{DAS (DER) RITSCHERT, SLOWENISCH RIČET ODER JEŠPRENJ}

Diese Speise ist von Legenden umgeben, gilt sie doch in Kärnten als bodenständiges, ${ }^{13}$ in Wien als jüdisches Gericht (vgl. Hornung 2002: 635 mit Lit., Kranzmayer 1949: 448, Wehle 1980: 241). Die historische Wirklichkeit ist jedoch eine andere: es handelt sich beim Ritschert um eine alte bairisch-österreichische Speise, die auch für die Bedürfnisse der jüdischen Küche adaptiert wurde (statt Schweinefleisch Geflügel, besonders Gänsefleisch) und die sich in Kärnten noch heute großer Beliebtheit erfreut und auch den Besuchern dieses Bundeslandes mundet. Der erste urkundliche Beleg der Speise (nicht des Wortes) stammt aus dem Jahre 1485, als dem Bischof von Caorle im Gailtal auf der

10 Slowenisch kvočni kommt von kłoca 'Klötze, Dörrbirne', entlehnt aus mittelhochdeutsch klōtze zu kärntnermundartlich khloatsn/khleatsn.

11 So auch im Slowenischen (palačinke); das Wort stammt aus dem Ungarischen und ist dort ein rumänisches Lehnwort aus plăcintă 'Pastete, Pfannkuchen' (aus latein. placenta). Näheres Bezlaj (1995: 5) u. Snoj (2003: 487f.).

12 Neben weiteren Bezeichnungen, s. die Karte in König (1996: 240).

13 „Ritschert gehört neben den Kasnudeln zu den am häufigsten anzutreffenden Kärntner Spezialitäten“" (Setz 1993: 52). 
Burg Khünegg (Khünburg bei Hermagor) „Gerste in fetter Fleischsuppe“ serviert wurde (Hornberg 1984: 239). ${ }^{14}$ Das Wort selbst begegnet zum ersten Mal im Klosterkochbuch von Tegernsee (1534) als ru(e)tschart 'gedämpfte Erbsen' (Hepp 1970: 216); Schmeller (1996: II 191) nennt es Rütscher, eine Speise aus gekochten Erbsen und Gerste oder aus Erbsen und Linsen. Alle mir zugänglichen Rezepte weisen (heute) als Hauptbestandteil Rollgerste mit Hülsenfrüchten (Erbsen oder Bohnen, auch Linsen) aus, wobei meist Geselchtes mitgekocht wird; die wichtigste Würze ist Liebstöckel, mundartlich Lust(st)ock, slowenisch luštek (vgl. Bezlaj 1982: 157, Striedter-Temps 1963: 173, Snoj 2003: 369 s. v. luštrek, Pleteršnik 2006: II 534 [neben luštək]); dazu die Redewendung „ohne Lustock kein Ritschert" (Miklau 1964: 42). ${ }^{15}$ Das Synonym dazu ist in Kärnten ursprünglich Gerstbrein, auch im Slowenischen ješprenj oder ješpranj [jéšprain] (so im Gailtal) aus deutsch-mundartlich Gerstbrein [geə(r)šprain]) neben ričet, gesprochen [ríčət], beides Lehnwörter aus dem Deutschen. Gerstbrein bedeutet eigentlich 'Rollgerste' (nicht -brei; deutsch Brei und Brein sind zwar etymologisch dieselben Wörter, doch sollte standarddeutsch Brei von mundartlich Brein (Prein) unterschieden werden, steht doch bei letzterem das Korn als Ausgangsprodukt semantisch im Vordergrund, vgl. Hepp 1970: 196f.). Dieses Wort ist also eine Benennung des „Ritscherts“ nach der Hauptzutat.

Die etymologische Deutung des Wortes Ritschert ist schwierig, da sowohl das grammatische Geschlecht als auch der Wortausgang schwanken. Für Kärnten notiert Lexer (1862: 209) ritschad(e) (das) 'gekochte und mit Erbsen vermengte Gerste', für Wien Hornung (2002: 635) Ritscha(d) (das/der) 'Gericht aus Gerste, Hülsenfrüchten (Erbsen, Bohnen oder Linsen) und teilweise Fleischstückchen (Selchfleisch, nach jüdischer Tradition Geflügel)', Schmeller (1996: II 191) Rütscher (der) 'Gericht aus Erbsen und Gerste', Hepp (1970: 216) Ru(e)tsch, Rutschart (der; -ue- wohl alte Schreibung für - $\ddot{u}$-, da nichts auf einen alten Diphthong hinweist), in der Steiermark Ritscher( $(t)$ und Ritschet; vgl. auch die Entlehnungen im Slowenischen ričet [-ə-] (Striedter-Temps 1963: 210, Bezlaj 1995: 178, Pleteršnik 2006: II 423.) und řiča im Tschechischen.

Als Ausgangswörter bieten sich entweder schwäbisch Rutsch 'Kachel (flaches, irdenes Kochgerät)' (Hepp 1970: 216 mit Lit.) oder die alte Nebenform zu rutschen, mittelhochdeutsch rütschen 'gleiten, rutschen' (Lexer 1983: 174) an. Im ersten Fall wäre der Name der Speise vom Kochgeschirr bezogen (vgl. auch ritschert 'Kartoffelpfannkuchen' in Hessen sowie Ritschering 'Pfanne' im Rotwelsch, so Hornung 2002: 635 mit Lit.) wie z. B. Pf(l)anzel aus Pfann-zelte oder Reindling zu Reine sowie Bezeichnungen wie Teller-fleisch, Kessel-gulasch, Suppen-topf usw. Im zweiten Fall käme der Name von der Eigenschaft der Ausgangsprodukte, die in Tat 'rutschen' bzw. 'gleiten', worauf ja auch das semantisch ähnliche bairisch-österreichische Roll-gerste hinweist. Offen muss allerdings der Auslaut bleiben; in Frage kommt -er, was mit dem männlichen Geschlecht übereinstimmt (das - $t$ wäre dann sekundär wie in mundartlich Senft, Teicht usw.) und zu beiden Ausgangswörtern passen würde. Wahrscheinlich handelt es sich wortbildungsmäßig um ein altes (sächliches)

14 Dort auch zwei Rezepte.

15 Rezepte für Kärnten s. Miklau (1964: 42, 26 u. 65), Angerer (1997: 98); weitere Rezepte Hornberg (1984: 239f.) und Maier-Bruck (1984: 97 u. 376), Kofranek (1975: 376); statt Geselchtem auch Gänsefleisch, letzteres v. a. bei den Juden beliebt. 
Kollektivum aus germanischer Zeit, althochdeutsch -idi, mittelhochdeutsch -ede (vgl. Meid 1967: 149f. mit vielen weiteren Beispielen), worauf die südbairischen Bildungen auf -ede/-ete zurückgehen (vgl. Hornung 1964: 73); dafür spricht auch das in Kärnten und der Steiermark allein übliche neutrale Geschlecht. Diese Ansicht wird durch die Einträge bei Lexer (1862: 209) und in anderen Wörterverzeichnissen sowie durch mehrere Parallelen bestätigt: ritschad(e) (das) 'gekochte und mit Erbsen gemengte Gerste' (Lexer 1862: 209), Rütschede (das) 'Gericht aus Gerste und Bohnen' (Villach, so Herzmansky-Kulterer 1969: 393). Daher möchte ich Ritschert als altes neutrales Kollektivum deuten, semantisch ganz ähnlich der bairisch-österreichischen (genauer:) süddeutschen Bezeichnung 'Rollgerste' (= 'rollende Gerste') als 'Rutschendes, Gleitendes' (Pohl 2004: 28ff.).

\section{MUNGGEN UND TALGGEN (ALTE BÄUERLICHE KOST SLAWISCHER HERKUNFT, ZU SLOWENISCH MOKA UND RUSSISCH TOLOKNÓ)}

Unter diesen beiden Bezeichnungen (auch Munken und Talken geschrieben) ${ }^{16}$ versteht man ein uraltes bäuerliches Vollkornnahrungsmittel (Miklau 1964: 130f.) ${ }^{17}$ - mit $\mathrm{Ha}$ fer. Lexer verzeichnet diese beiden Wörter als talk, talgge m. 'Hafergrütze' (Drautal, so Lexer 1862: 51) und munkn, munggn $\mathrm{f}$. 'eine Nationalspeise aus Hafer- und Gerstenmehl; (auch) kleines Stück Brot' (Feldkirchen, so Lexer 1862: 193). Hornung (1968: 181) verzeichnet die Munggn als Mölltaler Frühstücksspeise, genauer werden von ihr aber beide Wörter, die im Grunde genommen dieselbe Speise bezeichnen, so beschrieben (Hornung 1966: 174 u. 176): Munkn 'Altkärntner Nationalspeise aus Hafer-, Gersten- und Bohnenschrot, der gekocht, getrocknet und dann gemahlen aufbewahrt wird, um fallweise mit heißem Wasser angerührt zu werden'; Tålggn 'Hafer-, Gerstenund Bohnenschrot, gekocht, getrocknet und dann gemahlen', auch für zerkleinerte und getrocknete Birnen verwendet. Nach Miklau (1964: 130f.) findet man den Ausdruck Talggen v. a. im Nockgebiet, Munggen im Lesach- und Mölltal. ${ }^{18}$

16 Die traditionelle Schreibung $g g$ steht für nicht-affriziertes $k$, das im Normalfall entlehntes $/ \mathrm{k} /$ ersetzt.

17 In Tirol kommen die beiden Wörter in einer anderen Bedeutung vor, vgl. Schatz (1993: 438) mungge (f.) in Osttirol 'Brotbrocken', das Diminutiv minggile 'kleines Brot' (diese Bedeutung gibt auch Lexer 1862: 193 an) bzw. S. 626f. talgge, talggn (m.) 'teigige Masse, schlecht durchkochte Mehlspeise, unausgebackenes Brot'. Anders in der Steiermark: Dalken 'geröstetes Hafermehl in Milch zu dickem Brei verkocht und mit Butterschmalz serviert' (vgl. Hutterer/Kainz/ Walcher 1987: 260, ohne nähere Angaben bei Seebacher-Mesaritsch 1994: 41; die Munken werden dort S. 153 als Brei, der aus dem Mehl getrockneter Birnen hergestellt wird, beschrieben). Ein Hinweis auf die Steiermark auch bei Schmeller 1996: I 505). Im Salzburger Lungau sind die Munggen eine Speise aus gebrochenen Körnern in Schmalz geröstet (Ziller 1979: 123).

18 Von Miklau (1964: 130f.) wird auch die Herstellung beschrieben; verwendet wurden sie entweder warm aufgeweicht als Frühstücksspeise oder (ähnlich wie Reis) als Beilage, auch als Suppeneinlage. Sie können auch zu „Trippen“ („Talggentrippen“, S. 61 bzw. Maier-Bruck 1984: 106) weiterverarbeitet werden. Das Ausgangsgetreide par excellence war Hafer, wozu auch die slawischen Parallelen passen, die das hohe Alter der Speise unterstreichen, doch auch auf Roggen und andere Getreidemischungen wurde zurückgegriffen. - Unter Trippen (auch Gståmpftes) versteht man 'geschälte, gekochte und gestampfte Erdäpfel' (Miklau 1964: 159), nicht zu verwechseln mit Trip(p)a 'Kutteln, Kaldaunen' (aus dem Italienischen). 
Der Grundbestandteil der Tålggen ist Hafer (Rhamm 1909: 210f.); in seiner Monographie bringt Rhamm (1909: 211ff.) mehrere Varianten, so wurde Hafer allein verwendet in der Reichenau oder im Görtschitztal, in Feld am See wurden die „Habertalken" mit Bohnen, auch Erbsen, vermischt, in Kleinkirchheim waren 2/3 Hafer und 1/3 Feldbohnen üblich. Im Liesertal gab es zwei Arten von Talggen, die Piantalken 'Birntalggen' und Gampertalken $;{ }^{19}$ erstere wurden aus gedörrten Holzbirnen hergestellt, die man zerstampfte und zu Mehl mahlte (als Nudelfüllung und über Nockerln gestreut), letztere wurden aus Hafer, Gerste und Mais zu gleichen Teilen gemischt, gesotten, im Backofen getrocknet und schließlich gemahlen. Gegessen wurden sie eingerührt in süße (zum Frühstück) oder saure Milch (zum Nachtmahl), aber auch ähnlich zubereitet wie Plenten 'Polenta'.

Beide Wörter, Munggen wie Talggen, sind aus dem frühen Mittelalter überkommene Wörter slawischen Ursprungs. Das Wort Munggen ist ein frühslowenisches Lehnwort (aus altslawisch bzw. frühslowenisch *moka 'Mehl' mit Nasalvokal, heute slowenisch moka). Ein ebenso hohes Alter müssen die Talggen haben, die auf einem urslawischen *tălkŭnă (woraus russisch toloknó 'Art Hafergrütze', ins Finnische als talkkuna entlehnt, schon im finnischen Nationalepos Kalevala bezeugt, vgl. Rhamm 1909: 219) beruhen, was ein slowenisches *tlakno (vgl. polnisch ttokno 'Speise aus Hafermehl, heißem Wasser und Milch') ergeben hätte müssen. Wahrscheinlich handelt es sich um ein slawisches Erbwort, zur Wurzel *tălk- 'stoßen, (zer)stampfen' in russisch toloč'/tolkat', zur Bedeutung vgl. auch im Keltischen u.a. kymrisch („Welsh“) talch 'granum contritum’ ('geschrotetes/gemahlenes Korn') bzw. 'Bruchstück, Mahlkorn', altkornisch talch 'Kleie' (vgl. Trubačev-Vasmer 1973: 73 mit Lit. u. Pokorny 1959: I 1062)..$^{20}$ Das Wort muss also sehr früh ins Deutsche gelangt sein - spätestens zur Zeit der slawischen Liquidametathese, die in den slawischen Einzelsprachen zur Umstellung von *tălk- zu tlak-/tlok-/tolok- geführt hat, also etwa im 9. Jh. Die Talggen sind in Österreich weit verbreitet, außer in Kärnten und Osttirol auch im Salzburger Lungau und Teilen der Steiermark. ${ }^{21}$

19 Gamper nach Lexer (1862: 107) eine Speise aus Hafermehl., nach Miklau (1964: 155) 'Brei' (bzw. eine breiige Speise), wohl zu mittelhochdeutsch un-gamper 'steif' (das unbelegte gamper muss also das Gegenteil von 'steif' bedeutet haben).

20 Nach Kranzmayer (1949: 459), zuletzt WBMÖ IV 506, stamme das Wort letzten Endes aus dem Mongolischen und sei über die Awaren in unseren Bereich gelangt, was man zwar nicht ganz ausschließen kann, aber für diese frühe Zeit nicht sehr wahrscheinlich und darüber hinaus lautgeschichtlich bedenklich ist, wie übrigens auch die Ansicht, dass ungarisch tarhonya 'Art Teigreis, Eiergraupen' damit zusammenhängt (Kranzmayer 1949: 460, Rhamm 1909: 221 Anm.).

21 Davon zu trennen sind allerdings die Wiener (auch nieder- und oberösterreichischen) Dalken aus Germ- bzw. Hefeteig („Böhmische Dalken“), die auf einem anderen slawischen Wort beruhen (tschechisch dolek 'Vertiefung'), vgl. Kranzmayer (1949: 459), WBMÖ IV (505f.), zuletzt Hornung (2002: 221), Pohl (2007: 54). - Beide Wörter vermengt bei Schmeller (1996: I 505). - Rezepte bei Kofranek (1975: 315) u. Maier-Bruck (1984: 178). 


\section{STRANKERL 'FISOLE, GRÜNE BOHNE' (ZU FRÜHSLOWENISCH *STRĂNK-)}

Ähnlich wie die Munggen auf frühslowenisch *moka 'Mehl' (s. Kap. 8) beruhen, gehen auch die Strankerln (so meist geschrieben, genauer Stranggelein, ${ }^{22}$ Singular [štránkəle], Plural [štránkəlen/-lan]) auf eine frühslowenische Form *strănkъ bzw. strănkā '(Bohnen-) Schote' zurück, noch bevor der gemeinslawische Lautwandel $\breve{a}>\breve{o}$ bzw. die Nasalierung ăn/ŏn $>Q$ eingetreten war; im heutigen Slowenischen entspricht strok 'Hülse, Schote'. Die heute im Deutschen allein übliche Diminutivform setzt ein althochdeutsches *stranka $+-i l \bar{l}(n)$ voraus, das über mittelhochdeutsch *stränkilīn dann zu Stranggelein wurde (Pohl 2004: 84f.).

An Versuchen, das Wort als germanisch zu etymologisieren, hat es nicht gefehlt, so hat man es u. a. mit deutsch Strang 'Seil, Strick' in Verbindung gebracht (so Lexer 1862: 243, Schmeller 1996: II 1 817), was lautlich und semantisch äußerst unwahrscheinlich ist.

\section{SASAKA UND SLOWENISCH ZASEKA}

In letzter Zeit hat sich immer mehr die Bezeichnung Sasaka für 'Verhacket' (meist Verhackert geschrieben) verbreitet. Dieses ist ein beliebter Brotaufstrich, der aus geräuchertem und ausgelassenem „durchzogenem“ Speck, entsprechend gewürzt, hergestellt wird. Ursprünglich war dieses Wort nur im gemischtsprachigen Gebiet verbreitet, wird aber heute auch als Produktbezeichnung von Firmen verwendet. Sasaka beruht auf gleichbedeutendem slowenischen zaseka 'verhackter Speck', zu sekati 'hacken' (vgl. Pohl 2004: 81 u. 2007: 126 u. 151; Rezept u.a. bei Miklau 1997: 215).

\section{GAISLITZ (AUS SLAWISCH *KYSELICA)}

Die alte bäuerliche Speise Gaislitz findet sich bei Lexer (1862: 112, vgl. auch Hepp 1970: 206f.) unter dem Eintrag geislaz, -liz (m.) bzw. geislazn (f.) 'Speise aus Hafermehl' (Möll-, Drautal, Unterkärnten). Eine genaue Beschreibung dieser Speise bietet Hornung (1964: 79) für Kals (Osttirol), wo diese Speise gāi(z)litß lautet und ein saurer, fettloser Haferbrei ist, der in erstarrtem Zustand kalt gegessen wird. Laut Kranzmayer (1949: 448) ist diese Speise auch Bestandteil der Oberkärntner Bauernkost. Auch hier haben wir ein altes slawisches Lehnwort vor uns, das schon in mittelhochdeutscher Zeit belegt ist: gîs(e)litz(e) (m., f.) 'breiartige Speise' (Lexer 1983: 73) ${ }^{23}$ und auf slawisch *kyselica 'Säuerliches' beruht (so auch Hornung 1964: 79). Im südbairischen Raum ist eher von altem slowenischen kiselica (heute meist 'Sauerampfer') auszugehen, in anderen Gegenden auch von tschechisch kyselice 'Säuerliches' wie u. a. im Stift St. Florian bei Linz, wo diese Speise schon im 12. Jh.. als giselitz zitiert wird (vgl. Rhamm 1909: 215). Sie scheint weit verbreitet gewesen zu sein. Zwei Jahrhunderte später wird im Gedicht „Der Maier Helmbrecht“ von

22 Zur Schreibung gg s. o. Anm. 16.

23 Auch bei Schmeller (1996: I 952) als die Geislitze 'eine geringe Speise' / der Geislitz 'Haferbrei' enthalten. 
Wernher dem Gartenär aus der Gegend zwischen Wels und Treuenfels diese Speise als geyslitze überliefert (nach Rhamm 1909: 215). Wie bei den Tålggen gibt es auch bei dieser Speise einen Bezug zum Russischen. Dort wird zwar nicht die Form *kyselica verwendet, sondern kisél' (aus *kyselb), was nach dem Dahl'schen Wörterbuch ein Brei aus Hafer, Gerste und Weizenmehl ist, der warm gestellt und gesäuert wird. Urkundlich ist diese Speise bereits 997 belegt (Rhamm 1909: 220).

Bemerkenswert erscheint die Tatsache, dass die Wörter (und Sachen) Tålggen, Munggen und Gaislitz zwar eindeutig slawischer Herkunft sind, aber bei unseren heutigen slawischen Nachbarn nicht mehr vorkommen, wohl aber bei den Russen. Daraus kann man schließen, dass bei den alten Slawen der Hafer eine sehr große Rolle gespielt hat, was bei den Russen (und Finnen) im Osten noch lange erhalten geblieben ist und früher auch bei den Alpenslawen im Westen verbreitet war, noch vor der Zeit, als man in den Alpen deutsch sprach. Daher sind die Lautformen dieser Speisen auch aus slawistischer Sicht sehr altertümlich und sie werfen ein interessantes Licht auf die Kulturgeschichte des Essens und den deutsch-slawischen Sprachkontakt.

\section{TOPANITZ (ALTSLOWENISCH *TOPENICA) UND OBLITZEN (SLOWE- NISCH OBLICA)}

Zum Abschluss ein Blick nach Osttirol. Ein slowenisches Reliktwort in Kals (Osttirol) ist die Speise Topanitz, die Hornung (1964: 79) als wenig schmackhafte trockene Bähschnitten beschreibt und die auf ein altslowenisches *topenica (zu topel 'warm', topiti 'zum Schmelzen bringen') zurückgehen dürften. Ein weiteres slowenisches Reliktwort in Oberkärnten und Osttirol (Defereggen-, Iseltal) ist Oblitzen 'weiße Rübe' aus slowenisch mundartlich oblica 'gesottene oder gebratene Rübe' (vgl. Hornung 1964: 77 u. 159, Lexer 1862: 200).

\section{Literatur}

ANGERER, Tatjana (1997) Hausmannskost aus Südkärnten. Übers. von Alois Angerer. Klagenfurt/Ljubljana/Wien: Hermagoras/Mohorjeva.

ANGERER, Tatjana (1998) Koroška kuharica. Priljubljene domače jedi in pijače. Popravljen ponatis. Celovec: Mohorjeva.

BEZLAJ, France (1976) Etimološki slovar slovenskega jezika. Prva knjiga: A-J. Ljubljana: SAZU.

BEZLAJ, France (1982) Etimološki slovar slovenskega jezika. Druga knjiga: $K-O$. Ljubljana: SAZU.

BEZLAJ, France (1995) Etimološki slovar slovenskega jezika. Tretja knjiga: P-S. Dopolnila in uredila Marko Snoj in Metka Furlan. Ljubljana: SAZU.

BEZLAJ, France (2005) Etimološki slovar slovenskega jezika. Četrta knjiga: $\check{S}-\check{\text {. }}$ Hrsg. von M. Snoj/M. Furlan. Ljubljana: SAZU, Založba ZRC.

HORNBERG, Ulrike (1984) Österreichs Küche. Die klassischen Rezepte. Wien: Brandstätter.

HEPP, Eva (1970) „Die Fachsprache der mittelalterlichen Küche.“ In: H. Wiswe (Hrsg.), Kulturgeschichte der Kochkunst. München: Moos, 185-224. 
HERZMANSKY-KULTERER, Solveig (1969) Der alte Wortschatz der Bauern, Handwerker und Knappen in der Umgebung von Villach. Dissertation. Wien: Universität Wien.

HORNUNG, Maria (1964) Mundartkunde Osttirols. Wien: Österreichische Akademie der Wissenschaften.

HORNUNG, Maria (1966) „Mundartwörterverzeichnis.“ In: K. Dinklage/K. Erker/H. Prasch/F. Koschier (Hrsg.), Geschichte der Kärntner Landwirtschaft und bäuerliche Volkskunst Kärntens. Klagenfurt: Heyn, 169-177.

HORNUNG, Maria (1968) „Die Mundart des oberen und mittleren Mölltales.“ In: H. Prasch (Hrsg.), Um die Möll: Volkskunde eines Kärntner Tales. Spittal/Drau: Bezirksheimatmuseum, 178-189.

HORNUNG, Maria (2002) Wörterbuch der Wiener Mundart. Wien: öbv\&hpt.

HUTTERER, Claus Jürgen/Walter, KAINZ/Eduard, WALCHER (Hrsg.) (1987) Weststeirisches Wörterbuch. Wien/Köln/Graz: Böhlau.

KOFRANEK, Albert (1975) Die gute Wiener Küche. Wien: Kremayr \& Scheriau.

KÖNIG, Werner $\left({ }^{11} 1996\left[{ }^{10} 1994\right]\right) d t v-$ Atlas zur deutschen Sprache. München: dtv.

KRANZMAYER, Eberhard (1949) „Kärntner Bauernkost und ihre Geschichte.“ Carinthia I, 139, 446-462.

LEXER, Matthias (1998 [1862]) Kärntisches Wörterbuch. Vaduz: Sändig Reprints.

LEXER, Matthias (1983) Mittelhochdeutsches Taschenwörterbuch. 37. Auflage. Stuttgart: Hirzel.

MAIER-BRUCK, Franz (1984) Vom Essen auf dem Lande. Das große Buch von der österreichischen Bauernküche und Hausmannskost. Wien: Kremayr \& Scheriau.

MEID, Wolfgang (1967) Germanische Sprachwissenschaft III, Wortbildungslehre. Berlin: de Gruyter.

MIKLAU, Lia (1984) Kärntner Kochbüchel. 6. Auflage. Klagenfurt: Heyn.

PLETERŠNIK, Maks (2006) Slovensko-nemški slovar. I (A-O), II (P-Ž).Transliterierte Ausgabe. Hrsg. Metka Furlan. Ljubljana: ZRC.

POHL, Heinz Dieter (2004) Die Sprache der Kärntner Küche/Jezik koroške kuhinje. Klagenfurt/Celovec: Hermagoras/Mohorjeva.

POHL, Heinz Dieter (2007) Die österreichische Küchensprache. Ein Lexikon der typisch österreichischen kulinarischen Besonderheiten (mit sprachwissenschaftlichen Erläuterungen). Wien: Praesens.

POKORNY, Julius (1959) Indogermanisches etymologisches Wörterbuch. Bern/München: Francke.

RHAMM, Karl (1909) „Talken und Geislitz (russisch toloknó und kisélj), zwei alte slawische Hafergerichte." Carinthia I, 99, 209-222.

SCHATZ, Josef (1993 [1955/56]) Wörterbuch der Tiroler Mundarten. Innsbruck: Wagner.

SCHMELLER, Johann Andreas (1996 [1872/1877]) Bayerisches Wörterbuch. München: R. Oldenbourg.

SEEBACHER-MESARITSCH, Alfred (1994) Das Steirische Wörterbuch. Graz: Leykam. 
SEIBICKE, Wilfried (21983) Wie sagt man anderswo. Mannheim/Wien/Zürich: Dudenverlag.

SETZ, Helga (1993) Die Kärntner Küche. Klagenfurt: Heyn.

SNOJ, Marko (22003) Slovenski etimološki slovar. Ljubljana: Modrijan.

STRIEDTER-TEMPS, Hildegard (1963) Deutsche Lehnwörter im Slovenischen. Berlin/Wiesbaden: Harrassowitz.

TRUBAČEV, Oleg N. (1973) Ėtimologičeskij slovar' russkogo jazyka, tom IV. Überarb. Übers. von Max Vasmers Russischem etymologischen Wörterbuch. Moskva: Progress.

WBMÖ (1963) Wörterbuch der bairischen Mundarten in Österreich. Wien: Verlag der Österreichischen Akademie der Wissenschaften.

WEHLE, Peter (1980) Sprechen Sie Wienerisch. Wien: Ueberreuter.

ZEHETNER, Ludwig (2005) Bairisches Deutsch. Regensburg: edition vulpes.

ZILLER, Leopold (1979) Was nicht im Duden steht - Ein Salzburger Mundart-Wörterbuch. Salzburg: Gesellschaft für Salzburger Landeskunde.

\author{
Zusammenfassung \\ DEUTSCH-SLOWENISCHE WECHSELBEZIEHUNGEN \\ IN DER SPRACHE DER TRADITIONELLEN KÄRNTNER KÜCHE
}

Die Verschränkung der beiden Landessprachen in Kärnten zeigt sich nicht nur im Namengut, sondern auch in der Volkskultur, so auch in der Sprache der Küche. Viele Speisenbezeichnungen sind von der einen zur anderen Sprache ,gewandert" und sind somit gemeinsames Kulturgut. Insgesamt werden hier 16 Speisenbezeichnungen in beiden Sprachen vorgestellt und deren Herkunft und Etymologie im Lichte des Sprachkontaks erläutert. Darunter finden sich sehr frühe Entlehnungen aus dem Slowenischen ins Deutsche (z. B. Munggen und Talggen, aber auch relativ junge (z. B. Sasaka und Potitze). Auch manche deutsche Lehnwörter im Slowenischen weisen auf frühe Entlehnung hin (z. B. majželj und krapi). Hier zeigt sich die Relativität von Sprachgrenzen, da die jeweilige Volkskultur ein verbindendes Element zwischen den Sprechern beider Sprachen darstellt, wofür gerade das zweisprachige (Süd-)Kärnten ein gutes Beispiel ist - trotz aller historischer Turbulenzen.

Schlüsselwörter: Kärnten, Sprachkontakt, Volkskunde, Küche 


\section{Abstract \\ GERMAN-SLOVENIAN INTERACTION IN THE LANGUAGE OF THE TRADITIONAL CARINTHIAN KITCHEN}

The close interaction of the two national languages in Carinthia is not only evident in onomastic heritage, but also in the popular culture, as well as in the language of food and gastronomy. Many food names have "migrated" from one language to the other and are thus a common cultural property. This article presents 16 food names in both languages, explaining their origin and etymology in the light of language contact. Among them there are very early borrowings from Slovenian into German (for example, Munggen and Talggen), but also relatively recent ones (for example Sasaka and Potitze). Some German loanwords in Slovenian indicate an early instance of borrowing (for example majželj and krapi). In such cases, the relativity of linguistic borders becomes apparent, since a given folk culture can represent a connecting element between the speakers of both languages, as in the case of bilingual (southern) Carinthia, despite its turbulent history.

Keywords: Carinthia, language contact, ethnology, gastronomy

\section{Povzetek \\ NEMŠKO-SLOVENSKI JEZIKOVNI VPLIVI V JEZIKU TRADICIONALNE KOROŠKE KUHINJE}

Prepletenost obeh deželnih jezikov na Koroškem se ne kaže samo v lastnih imenih, temveč tudi v narodni kulturi, med drugim tudi v jeziku kulinarike. Iz enega $\mathrm{v}$ drug jezik je bilo prevzetih mnogo poimenovanj jedi, ki so sedaj del skupne kulturne dediščine. Skupno gre za 16 poimenovanj, predstavljena sta njihov izvor in etimologija v luči jezikovnega stika. Med nemškimi besedami je mogoče najti zelo zgodnje izposojenke iz slovenščine (npr. Munggen in Talggen), a tudi novejše (npr. Sasaka in Potitze). Zgodaj prevzeto je bilo tudi mnogo nemških izposojenk v slovenščini (npr. majželj in krapi). Ravno tukaj pa se pokaže relativnost jezikovnih meja, saj je neka narodna kultura povezovalni element med govorci obeh jezikov, za kar je - kljub zgodovinskim turbulencam - zgled ravno dvojezična (južna) Koroška.

Ključne besede: Koroško, jezikovni stik, etnologija, kuhinja 